Notas Clínicas

\title{
Osteosarcoma meníngeo sin exposición previa a radiaciones. A propósito de un caso
}

\author{
D. Márquez Medina*, M. I. Sáez Medina**, J. D. Cumplido Burón, J. A. García García*, \\ M. C. Talavera Hernández***, I. Blancas López-Barajas*, J. L. García Puche***
}

\section{Resumen}

Los osteosarcomas intracraneales primarios son lesiones verdaderamente infrecuentes. La radiación previa ha sido, descrita como un factor predisponente para el desarrollo de osteosarcomas meníngeos primarios. Presentamos el caso de un paciente diagnosticado de osteosarcoma meningeo primario sin exposición previa a radiaciones, tratado con cirugía, quimio y radioterapia, aprovechando para revisar el estado de la literatura al respecto.

\section{Palabras clave:}

Cirugía. Osteosarcoma meningeo primario. Quimioterapia. Radioterapia. Tratamiento.

Oncología, 2004; 27 (2):95-98

\section{Summary}

Primary intracraneal osteosarcomas are very rare. Previous radiation has been described as a predisposing factor to develop primary meningeal osteosarcomas. We report the case of a male patient diagnosed of primary meningeal osteosarcoma without previous irradiation history. He was treated by surgery, chemotherapy and radiotherapy. We made a review of the literature.

Key words: Primary meningeal osteosarcoma. Chemotherapy. Radiotherapy. Surgery.

* F.E.A. de Oncología Médica

** M.I.R. de Oncología Médica

*** Jefes de Sección y Servicio

Unidad integral de Oncología y Servicio de Anatomía Patológica. Hospital San Cecilio. Granada 


\section{Márquez Medina y cols.}

\section{Introducción}

Los sarcomas son neoplasias originadas en el tejido mesenquimal y que, por tanto, pueden presentarse en cualquier localización. Los osteosarcomas son sarcomas originados a partir de tejidos óseos, principalmente en la metáfisis de huesos largos, pero que pueden aparecer en huesos planos y, rara vez $(4 \%)$, en tejidos blandos ${ }^{1}\left(1^{\prime} 2 \%\right.$ de los sarcomas de tejidos blandos). Los sarcomas intracraneales primarios no originados en el cráneo son poco frecuentes pero condrosarcomas, mixomas, tumores fibrosos y sarcomas de Ewing han sido comunicados. Los osteosarcomas meníngeos primarios (OMP) son extremadamente raros ${ }^{2}$.

Los antecedentes de radiación están reconocidos como un factor de riesgo para el desarrollo de sarcomas en tejidos blandos y hueso ${ }^{3}$, y la radioterapia (RT) craneal ha sido descrita como un factor predisponente al desarrollo del OMP4. El intervalo entre la exposición a la radiación y el diagnóstico del osteosarcoma ronda los 16 años, con un rango que va de 4 a 35 años $^{5}$. El OMP ha sido también descrito meses después de la radiación ${ }^{6}$ y sin evidencia de ésta ${ }^{8,9}$.

En la literatura revisada, el OMP ha sido fundamentalmente descrito en pacientes varones de 35 a 56 años. Principalmente aparecen en pacientes tratados con RT por otros tumores (adenomas pituitarios, glioblastomas o meningiomas) $4,6,10$. Sus síntomas son similares a los de otras neoplasias intracraneales: Cefaleas, déficit locomotor progresivo o del campo visual, cambios de comportamiento, vómitos o inestabilidad, con una escasa incidencia de convulsiones. La exploración neurológica puede revelar distintos signos como hiperreflexia, dificultad de equilibrio o déficit sensitivo y locomotor.

Las exploraciones complementarias incluyen hemograma y bioquímica general y radiología de tórax, (generalmente normales), tomografía computerizada (TAC) o resonancia nuclear magnética cerebral (RNMC), que suelen mostrar masas hiperdensas, total o parcialmente calcificadas, con edema alrededor, y gammagrafía con ${ }^{99 \mathrm{~m} T e c n e c i o ~ y ~ r a d i o l o g i ́ a ~ o ́ s e a ~ c o m-~}$ pleta, a fin de garantizar un origen no metastásico del proceso.

El hallazgo patológico más común es la presencia de una matriz osteoide que contiene calcio y células atípicas uni o multinucleadas, poligonales o fusiformes, con un importante pleomorfismo nuclear, nucleolos prominentes y frecuentes mitosis atípicas. Esta matriz puede teñirse con pentacromo de Movat, anilina azul de Heidenhain y tinción de Von Kossa ${ }^{11}$.

La cirugía suele representar el tratamiento inicial, y en ocasiones único, del OMP. Una resección completa debe ser la meta de la cirugía, evitando biopsias previas por el riesgo de diseminación a lo largo de su recorrido y garantizando unos márgenes de seguridad convenientes.

El tratamiento adyuvante incluye RT, quimioterapia (QT) o ambas'. La QT basada en esquemas de doxorrubicina y cisplatino es la más frecuente (p.e. adriamicina, ifosfamida y cisplatino o ifosfamida, etopósido y cisplatino alternando con ifosfamida, etopósido y adriamicina) y efectiva incluso en esquemas de rescate para OMP metastásico ${ }^{10}$.

El OMP es una neoplasia de mal pronóstico que debe tratarse agresivamente combinando estrategias. La supervivencia sin recaída más allá de los dos años de seguimiento puede lograrse con una cirugía amplia y total, CT y RT' ${ }^{1}$.

\section{Caso clínico}

Presentamos el caso de un varón de 59 años que consulta por un cuadro de déficit intelectual, convulsiones, bradipsiquia, desorientación y estupor en los días previos. La TAC sin contraste muestra una lesión frontal izquierda periférica y de $3^{\prime} 5 \mathrm{cms}$ de diámetro, con una amplia base y efecto masa, acompañada de hernia trans-subcifoidal, colapso del tercer ventrículo y dilatación de los ventrículos laterales. Estos hallazgos fueron interpretados como la presencia de un menigioma del lóbulo frontal izquierdo y se efectuó una resección en Diciembre del 2000. La anatomía patológica fue informada como osteosarcoma meníngeo (Macros-

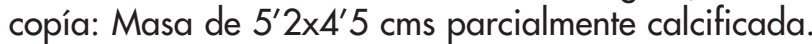
Microscopía: Tumor osteogénico bien delimitado, sin infiltración del parénquima. Trabéculas óseas irregulares conteniendo áreas atípicas, áreas bien diferenciadas con células osteoclásticas, necrosis y hemorragia).

La gammagrafía con ${ }^{99 m}$ Tecnecio fue positiva en el campo quirúrgico, los análisis de laboratorio normales y la TAC corporal mostraba parálisis del hemidiafragma izquierdo e hidrotórax.

Derivado a nuestra unidad y descartada la afectación metastásica, el paciente inició un programa de QT adyuvante, pero tras un primer ciclo de cisplatino y adriamicina, la resonancia nuclear magnética evidenció la persistencia de una neoplasia meníngea frontal rodeada de una colección de sangre, con efecto masa y colapso ventricular.

Se realizó una segunda resección con márgenes amplios y el patólogo informó en la pieza la persistencia de un osteosarcoma de la meninge dural, con infiltración del tejido cerebral y del hueso. Se programó un régimen de QT adyuvante con ciclofosfamida 600 
$\mathrm{mg} / \mathrm{m}^{2}$, bleomicina $15 \mathrm{mg} / \mathrm{m}^{2}$ y actinomicina-D 0'6 $\mathrm{mg} / \mathrm{m}^{2}$ (día $1 / 21$ días) y metotrexate semanal, que el paciente interrumpió por voluntad propia tras el primer ciclo. Por ese motivo se le administró RT adyuvante de Marzo a Mayo del 2000, $50+14$ Gy (5x2 Gy/semanales) de ${ }^{60}$ Cobalto.

El paciente continuó revisiones hasta Mayo del 2003, cuando se descubrió una recaída local mediante RNM, y fue enviado al servicio de Neurocirugía del Hospital Carlos Haya de Málaga. El 11-05-2003, refiriendo déficit intelectual e incontinencia urinaria, se le realizó una nueva RNMC que mostraba un proceso expansivo frontoparietal, extendido al lóbulo frontal derecho y al cuerpo calloso, y una TAC torácica que apreciaba múltiples nódulos metastásicos en ambos pulmones. Se descartó tratamiento con cirugía, QT y RT, y se administraron corticoides a altas dosis, consiguiendo una mejoría inicial.

Sin embargo, el 30 de Agosto del 2003, el paciente acudió a las urgencias de nuestro centro, presentando un severo deterioro de conciencia, con un índice de Karnoffsky del $30 \%$, disnea y trombosis del sistema femoral izquierdo, apreciándose en la placa de tórax múltiples metástasis pulmonares.

Conseguida su estabilización, se desestimó la aplicación de tratamiento oncológico activo y el paciente fue remitido a cargo de nuestra Unidad de Cuidados Paliativos.

\section{Discusión}

La comunicación de este caso está motivada por la rareza de encontrar un sarcoma osteogénico en un tejido sin hueso como son las meninges. El OMP es una neoplasia realmente infrecuente, cuyo tratamiento definitivo aún es controvertido debido a la escasez de casos conocidos y a la ausencia de estudios comparativos.

La necesidad de una cirugía radical con márgenes amplios es evidente. El riesgo de recaída local en sarcomas óseos y de tejidos blandos es alto cuando los márgenes quedan demasiado cerca del área tumoral, pero la presencia de estructuras cerebrales dificulta la cirugía en el OMP. Nuestro caso presentó un recaída local incluso con un informe patológico de resección completa y una segunda cirugía con amplios márgenes fue indispensable para el control del proceso.

En esta segunda intervención se apreciaba infiltración de las estructuras cerebrales y del hueso, y aunque a veces el OMP ha sido descrito como un tumor poco agresivo localmente, este hallazgo refuerza la importancia de una cirugía radical y amplia.
La efectividad de la QT adyuvante no pudo probarse dada la negativa del paciente a realizarla, aunque queda patente en la literatura revisada, como no pudo valorarse la eficacia de una QT paliativa. En este sentido, se emplearon dosis de RT similares a las descritas por otros autores. La infiltración de tejidos vecinos, la persistencia de tumor viable en la segunda resección y el riesgo de diseminación por los campos quirúrgicos debido a las reiteradas craneotomías fueron razones decisivas para la indicación de RT adyuvante.

Respecto al origen del OMP, la radioexposición previa ha sido ampliamente asumida como un factor predisponente para el desarrollo de sarcomas. Se conocen casos de OMP no irradiados, 10, lo que, como en el caso de nuestro paciente, nos llevan a pensar que la patogénesis del OMP no está aún completamente aclarada y que deben existir otros factores etiológicos que aún desconocemos.

Finalmente, podemos concluir que el OMP es un infrecuente sarcoma osteogénico originado en las meninges, que precisa de un tratamiento agresivo que combine cirugía, QT y RT para mejorar la supervivencia de los pacientes.

\section{Correspondencia:}

Dr. D. Márquez Medina

Secretaría de Servicio de Oncología Médica y Radioterápica Hospital Clínico Universitario San Cecilio de Granada

Avda. Doctor Olóriz, 16

E-18012 Granada

E-mail: dmarmed@hotmail.com

\section{Bibliografía}

1. Bonilla F, Provencio M, Salas C, España P. Primary osteosarcoma of the meninges. Ann Oncol 1994; 5: 965-6.

2. Bane BL, Evans HL, Ro JY et al. Extraskeletal osteosarcoma: A clinicopathologic review of 26 cases. Cancer 1990; 60: 2762-70.

3. Wiklund TA, Blomquist CP, Raty J et al. Postrradiation sarcoma. Analysis of a nationwide cancer registry material. Cancer 1991; 68: 524-31.

4. Osipov V, Ho KC, Krouwer HG, Meyer G, Shidham VB. Post-radiation desdifferentiation of meningioma into os- 
D. Márquez Medina y cols.

teosarcoma. BMC Cancer 2002; 2: 34.

5. Chung EB, Enzinger FM. Extraskeletal osteosarcoma Cancer 1987; 60: 1132-42.

6. Couldwell WT, Scheithaver BW, Rice SG, Zhang W and Stillerman CB. Osteosarcoma of the meninges in association with glioblastoma. Acta Neurochirurgica 1997; 139: 684-689.

8. Setzer M, Lang J, Turowski B, Marquardt G. Primary meningeal osteosarcoma: case report and review of the literature. Neurosurgery 2002; 51: 488-92.

9. Kachhara R, Nair S, Sandhyamani S, Bhattacharya RN. Primary osteogenic sarcoma involving sella-sphenoid sinus-case report. Neurol Med Chir 1999. 39 : 534-8.

10. Carpentier AF, Chantelard JV, Henin D, Poisson M. Osteosarcoma following radiation treatment for meningioma: report of a case and effective treatment with chemotherapy. J Neurooncol 1994; 21: 249-53.

11. Ringenberg MA, Neitzel LE, Zachary JF. Meningeal oste- 